\title{
Unchecked thrombin is bad news for troubled arteries
}

\author{
Eric Camerer
}

Cardiovascular Research Institute, UCSF, San Francisco, California, USA.

\begin{abstract}
Thrombin is clearly a key trigger of thrombosis, the proximal cause of most morbidity and mortality in atherosclerotic cardiovascular disease. Might thrombin also contribute to longer-term, structural changes in the arterial wall that promote narrowing and clotting? A study in this issue of the JCI argues that it can. Aihara et al. report that haploinsufficiency of heparin cofactor II, a glycosaminoglycan-dependent thrombin inhibitor, exacerbates injury- or hyperlipidemia-induced arterial lesion formation in mice, possibly by excessive thrombin signaling through protease-activated receptors (see the related article beginning on page 1514).
\end{abstract}

\section{Thrombin generation}

As the main effector protease of the coagulation cascade (Figure 1), thrombin is required for hemostasis in response to injury. Thrombin is generated when tissue factor $(\mathrm{TF})$, a transmembrane protein, binds plasma coagulation factors (1). Normally, TF is found mainly on cells in the extravascular compartment, and disruption of vascular integrity upon injury allows plasma coagulation factors to interact with TF. Factor VIIa complexes with TF to activate factor X, either directly or via factor IX activation, and the protease $\mathrm{Xa}$ converts prothrombin to thrombin. Active thrombin then cleaves fibrinogen to fibrin and triggers platelet activation and a variety of other cellular responses via $G$ protein-coupled protease-activated receptors (PARs). Four main inhibitors keep thrombin generation and activity in check: TF pathway inhibitor (TFPI), antithrombin (AT, also known as ATIII), heparin cofactor II (HCII), and thrombomodulin (TM) through activated protein $\mathrm{C}$ (APC). Imbalances in this system can have important consequences, as evidenced by the multiple human genetic diseases associated with loss of coagulation factor or inhibitor function.

Nonstandard abbreviations used: APC, activated protein C; AT, antithrombin; DS, dermatan sulfate; GAG, glycosaminoglycan; HCII, heparin cofactor II; HS, heparan sulfate; PCI, percutaneous coronary intervention; TF, tissue factor; TFPI, TF pathway inhibitor; TM, thrombomodulin.

Conflict of interest: The author has declared that no conflict of interest exists.

Citation for this article: J. Clin. Invest. 117:1486-1489 (2007). doi:10.1172/JCI32473.

\section{Pathological thrombin generation may have both acute and cumulative effects on arterial patency}

In atherosclerotic plaques and injured arteries, TF expression is induced in smooth muscle cells and macrophages and is thus enriched and more proximal to the vessel lumen than in normal arteries (2). Thus even superficial injury, whether spontaneous or induced by percutaneous coronary intervention (PCI), exposes TF, lipid surfaces, and matrix proteins to circulating coagulation zymogens and platelets, triggering thrombin generation and platelet activation (Figure 2). In the short term, this leads to formation of thrombi that may or may not grow to a size sufficient to cause symptoms. In the long term, repeated or continuous exposure of endothelial cells, smooth muscle cells, and tors, fibrin, and platelet-released products might contribute to expansion of arterial lesions. Similar mechanisms might promote restenosis and/or thrombosis after stent placement, where compromised reendothelialization may result in prolonged exposure of TF to plasma.

How might exposure of mural cells to coagulation proteases promote atherosclerosis and neointima formation after arterial injury? Coagulation is one of the first responses to tissue injury, and coagulation proteases can regulate cellular behaviors through PARs (3). Thrombin activates PAR1 and PAR4; upstream proteases (VIIa and Xa) can activate PAR1 and PAR2. Responses to PAR signaling in cell culture hint that coagulation proteases and PARs macrophages to active coagulation fac- together may help orchestrate not only hemostasis but also inflammation and repair after injury (4). Analogous to TF, both PAR1 and PAR2 are highly expressed in human atheroma relative to normal arteries and are induced in the arterial wall in response to injury in animal models (5). It is thus conceivable that hyperactivation of and hyperresponsiveness to coagulation proteases in the setting of arterial injury leads to excessive and therefore pathogenic repair responses (Figure 2).

A growing body of evidence from animal models supports a causal role for coagulation proteases and PAR signaling in arterial disease (Figure 1). Recombinant inhibitors (6-8) or PAR1 antagonists (9) ameliorate whereas anticoagulant deficiencies exacerbate (10-12) pathologic remodeling. Lack of effects of the anticoagulant heparin (13) and variable responses to another anticoagulant - hirudin (6) - in these models may point to roles for upstream proteases (6) or subtleties in anticoagulant function (discussed below). The study by Aihara et al. (14) in this issue of the JCI adds another piece to this puzzle with possible promise for a novel treatment strategy.

\section{Anticoagulant activity of $\mathrm{HCII}$}

Until recently, HCII has been in the shadow of AT. Like AT, HCII is a serpin whose activity increases several orders of magnitude in the presence of heparin or heparan sulfate (HS) glycosaminoglycans (GAGs) (15). Yet HCII is limited to thrombin in specificity and is not only less abundant than AT but also both inferior in basal antithrombin activity and a secondary player in heparinmediated anticoagulation. In mice, absence of AT, TFPI, TM, or APC results in coagulopathy and pre- or perinatal lethality (16), whereas HCII-knockout animals reach old age without signs of spontaneous thrombosis (17). Consistent with these findings, HCII deficiency does not appear to predict thrombophilia in humans (16).

What, then, is HCII's raison d'être? Photochemically-induced arterial injury did uncover a hyperthrombotic phenotype in 


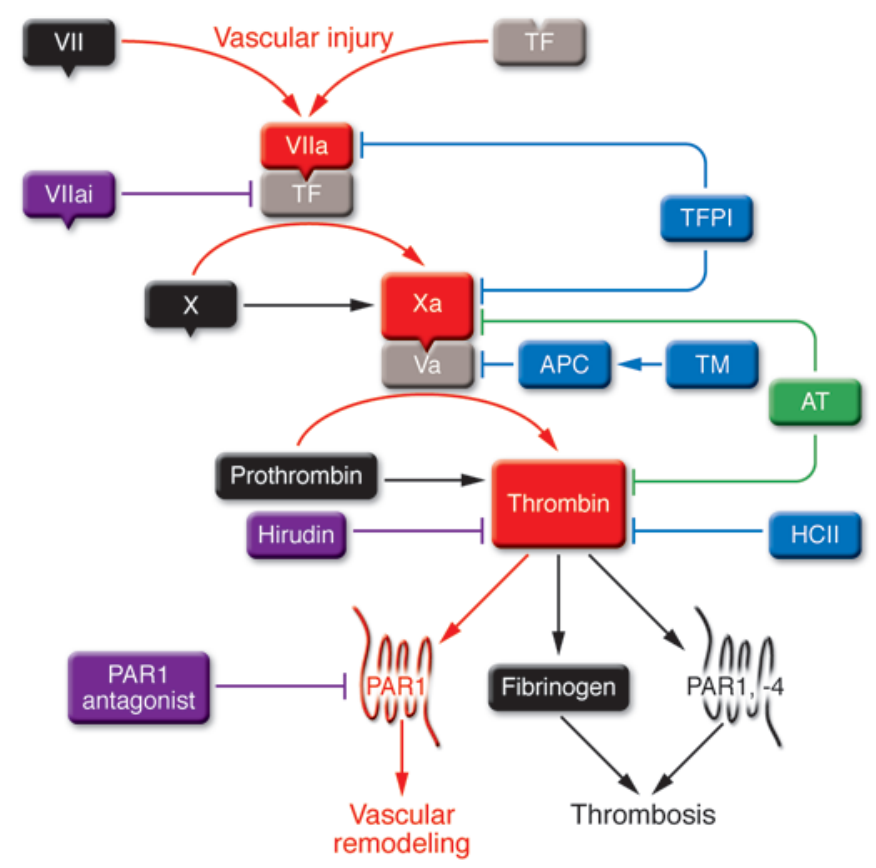

Figure 1

Perturbing the inhibitor balance has uncovered a possible role for thrombin signaling in vascular remodeling. Vascular injury may activate the coagulation cascade (only the extrinsic cascade is shown) by allowing plasma factor VII to interact with its extravascular cofactor TF. The TF:VIla complex activates factor $\mathrm{X}$, and $\mathrm{Xa}$ converts prothrombin to thrombin with the help of cofactor factor Va. Thrombin not only triggers thrombosis by cleaving fibrinogen and activating platelets through PARs (PAR4 in mice, PAR1 and PAR4 in humans), but also activates mural cells and leukocytes through PAR1. Partial loss (by haploinsufficiency of TFPI, or HCIl in the current study by Aihara et al.; ref. 14) or interruption (by APC resistance induced by the presence of factor V Leiden) of natural inhibitor function is associated with enhanced pathologic remodeling in animal models; AT appears to be an exception. Conversely, recombinant anticoagulant therapy (e.g., use of VIlai, TFPI, TM, or hirudin) reduces remodeling, as does loss or inhibition of the thrombin receptor PAR1. This suggests that thrombin contributes to pathologic remodeling and may imply a direct pathway from TF exposure to vascular injury through PAR1 signaling (red) (14), although other effectors of thrombin or upstream coagulation factors may also contribute to this process. VIlai, active site-inhibited VIla.

HCII-knockout mice (17), so HCII function does complement that of other anticoagulants. The key appears to be location; notable recent advances in HCII biology have come from studies of its tissue-specific activation (15). A unique feature of HCII is that, unlike AT, it can be activated by dermatan sulfate (DS) as well as HS GAGs. DS is enriched in areas low in anticoagulant HS such as the placenta and in the arterial wall; it may thus allow HCII to function where AT comes up short. HCII levels increase to $150 \%$ of normal during pregnancy but are $50 \%$ of normal in severe preeclampsia, suggesting a role for HCII in regulating placental hemostasis. DS appears to be the major anticoagulant GAG in the vessel wall (15), and unlike heparin, DS has been shown to inhibit smooth muscle hyperplasia in animal models $(13,15)$. Thus, a picture emerges where DS distribution may afford HCII a unique role in regulating extravascular thrombin activity after arterial injury (Figure 2). DS is modified and less able to support HCII activity in atherosclerotic plaques, so loss of HCII activity has been proposed as a possible reason why thrombin is left unchecked in this setting (15).

\section{HCll deficiency}

In their study in this issue of the JCI, Aihara et al. report (14) on an independently generated HCII-knockout mouse. Contrasting the original report (17), homozygous deficiency was not compatible with life. The reason for the discrepancy is elusive; the original study established absence of circulating HCII antigen and activity, and both knockout alleles were inbred to the same strain background. Aihara et al. did not assess survival in a mixed-strain background, so it is conceivable that they lost a vital modifier retained in the original knockout. They confirmed $5^{\prime}$ and $3^{\prime}$ recombination, single integration, and unaltered expression of neighboring genes. The authors suggest that the original allele may have been hypomorphic, but evidence is inconclusive. Whether embryonic lethality supports a role for HCII in regulating placental hemostasis or suggests that HCII has other targets than thrombin was not assessed in this study.

Addressing the role of HCII in arterial homeostasis in adult mice, Aihara et al. (14) showed increased hyperplasia after cuff- or wire-induced arterial injury in their heterozygous survivors. Effects were striking as HCII activity was reduced by the mere $40 \%$. Neointima formation increased with or without increased adventitial thickening, depending on the model; evidence of enhanced cell proliferation in these regions supported the outcome. Phenotype correction with purified human HCII provided elegant proof of specificity. Underscoring a critical role for HCII in regulating arterial homeostasis, the authors went on to show increased susceptibility to diet-induced atherosclerosis in an apoE-null background. HCII haploinsufficiency was associated with increased expression of PAR1 and induction of inflammatory and mitogenic gene products, some known to be regulated by PAR1, in remodeled lesions. Notably, the substantive findings in this study have been corroborated by Tollefsen and colleagues (15). Collectively, these data support a model where uninterrupted thrombin activity may promote inflammatory cell recruitment and smooth muscle expansion, possibly through PAR1 signaling (Figure 2).

\section{Perspectives}

A number of caveats regarding interpretation and extrapolation of the current study (14) should be mentioned. While decreased HCII levels may indeed yield increased thrombin activity, the report does not address the possibility that the effect of decreased HCII levels is due to dysinhibition of other proteases. While mutations that cause gain of function for thrombin might increase neointima formation, it does not necessarily follow that loss of function for thrombin would decrease formation. In this regard it is interesting to note that large doses of exogenous HCII did not reduce neointima formation in HCII-deficient mice beyond 


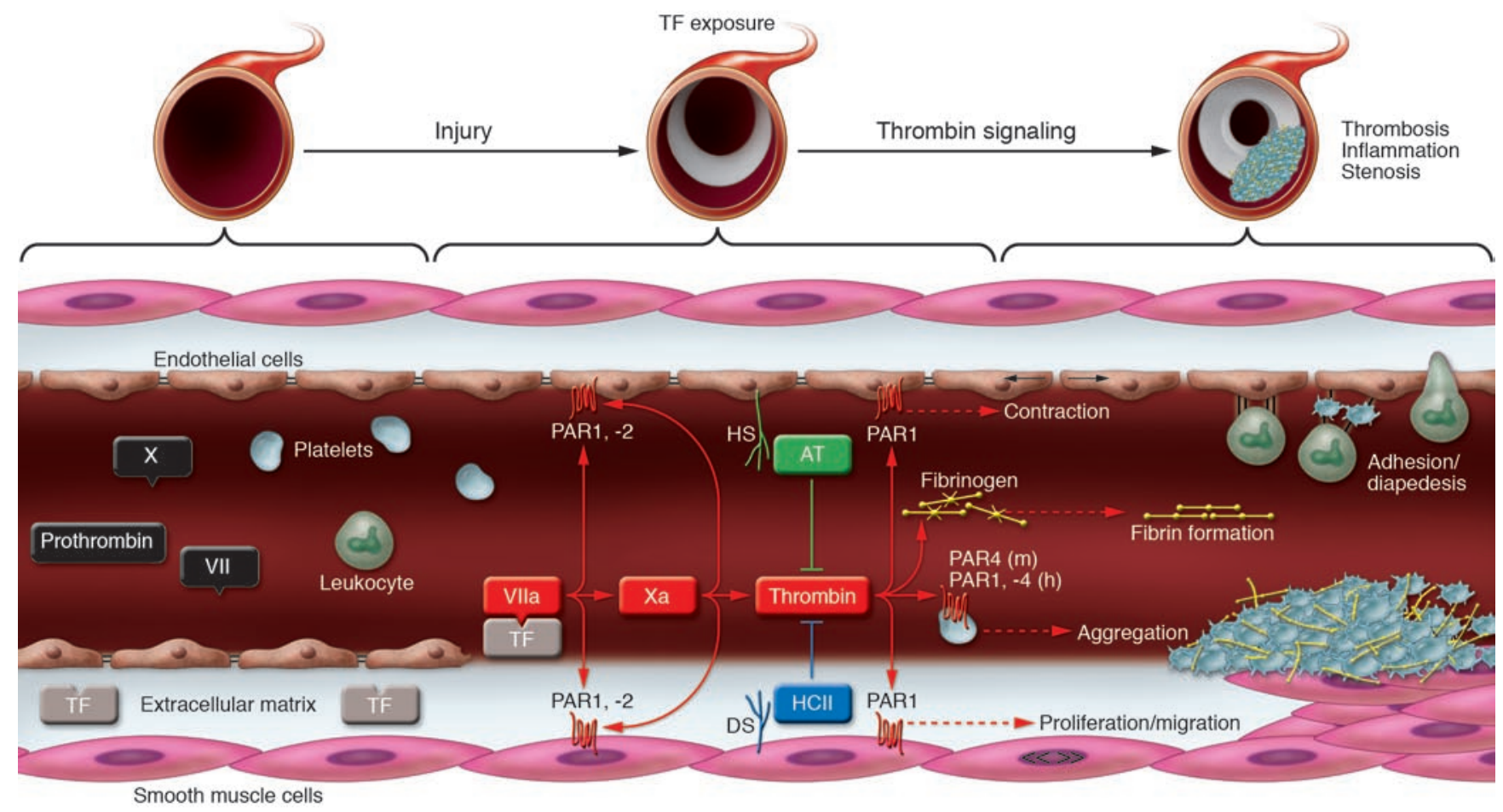

\section{Figure 2}

How might $\mathrm{HCll}$ deficiency exacerbate responses to arterial injury? Arterial injury may trigger thrombin formation by exposing extravascular TF to circulating coagulation zymogens (see Figure 1). Thrombin activates platelets and converts fibrinogen to fibrin, thus triggering thrombosis and thus, potentially, acute vascular occlusion. Both thrombin and upstream proteases VIla and Xa may also contribute long-term to arterial remodeling and narrowing (stenosis) by signaling to circulating and mural cells through PARs. At least in vitro, PAR activation triggers leukocyte chemotaxis, endothelial contraction/secretion/expression of adhesion molecules, and smooth muscle cell proliferation/migration. The thrombin inhibitor HCII may be uniquely suited to inhibiting thrombin in the vessel wall, since it is activated by DS, which is synthesized by smooth muscle cells and fibroblasts. AT, by contrast, is only activated by HS enriched in the subendothelial matrix. Tissue-specific thrombin inhibition may thus explain why even partial loss of $\mathrm{HCll}$ activity exacerbates arterial remodeling in response to injury or hyperlipidemia in mice, as shown by Aihara et al. in this issue of the $\mathrm{JCl}(14)$. m, mice; h, humans.

levels in wild-type controls. While PARs are reasonable candidates for mediating effects of increased thrombin generation, dependence on PARs was not demonstrated. And importantly, rodent models of neointima formation have been poor predictors of success for new strategies aimed at preventing restenosis after PCI in humans. That said, the current study is provocative in the context of recent findings and opportunities. While whether HCII deficiency is a risk factor for atherosclerosis in humans is debatable $(15,18)$, two recent clinical studies demonstrated an inverse correlation between HCII deficiency and restenosis after PCI (19, 20). A prerequisite for HCII therapy to be effective may be added correction of the functional deficiency of DS in atheroma. Exogenous DS is distributed to the extravascular space, and DS treatment alone has been shown to reduce restenosis in animal models $(13,15)$. As DS does not activate AT, DS therapy might correct a defect specific to the atheroma and thus provide local anticoagulation without perturbing normal hemostasis. An alternative strategy to target this pathway may be through PARs $(3,5,21)$. As human platelets carry PAR4 as a low-affinity "backup" receptor for thrombin, PAR1 antagonism might limit thrombosis and most cellular actions of thrombin while retaining fibrin formation and enough residual platelet activation to prevent bleeding. An oral PAR1 antagonist evaluated against acute thrombosis after PCI did not increase bleeding in a recent phase II clinical trial (22); if it proves efficacious in preventing acute events, this antagonist might also be beneficial to efforts toward establishing long-term patency.

\section{Acknowledgments}

The author is grateful to Shaun Coughlin for suggestions and insightful comments.
Address correspondence to: Eric Camerer, University of California, San Francisco, CVRI, 600 16th Street, MC 2240, San Francisco, CA 94158-2517, USA. Phone: (415) 476-6172; Fax: (415) 476-8173; E-mail: eric. camerer@ucsf.edu.

1. Colman, R.W., Clowes, A.W., George, J.N., Goldhaber, S.Z., and Marder, V.J. 2006. Overview of hemostasis. In Hemostasis and thrombosis: basic principles and clinical practice. R.W. Colman, J. Hirsh, V.J. Marder, A.W. Clowes, and J.N. George, editors. Lippincott, Williams \& Wilkins. Philadelphia, Pennsylvania, USA. 3-16.

2. Taubman, M.B., et al. 1997. Tissue factor in the pathogenesis of atherosclerosis. Thromb. Haemost. 78:200-204.

3. Coughlin, S.R. 2000. Thrombin signalling and protease-activated receptors. Nature. 407:258-264.

4. Coughlin, S.R., and Camerer, E. 2003. PARticipation in inflammation. J. Clin. Invest. 111:25-27. doi:10.1172/JCI200317564.

5. Major, C.D., Santulli, R.J., Derian, C.K., and Andrade-Gordon, P. 2003. Extracellular mediators in atherosclerosis and thrombosis: lessons from thrombin receptor knockout mice. Arterioscler. Thromb. Vasc. Biol. 23:931-939.

6. Jang, Y., et al. 1995. Influence of blockade at spe- 
cific levels of the coagulation cascade on restenosis in a rabbit atherosclerotic femoral artery injury model. Circulation. 92:3041-3050.

7. Li, Y.H., et al. 2006. Thrombomodulin plays an important role in arterial remodeling and neointima formation in mouse carotid ligation model. Thromb. Haemost. 95:128-133.

8. Sarembock, I.J., et al. 1991. Effectiveness of recombinant desulphatohirudin in reducing restenosis after balloon angioplasty of atherosclerotic femoral arteries in rabbits. Circulation. 84:232-243.

9. Derian, C.K., Maryanoff, B.E., Zhang, H.C., and Andrade-Gordon, P. 2003. Therapeutic potential of protease-activated receptor-1 antagonists. Expert Opin. Investig. Drugs. 12:209-221.

10. Singh, R., et al. 2003. Tissue factor pathway inhibitor deficiency enhances neointimal proliferation and formation in a murine model of vascular remodelling. Thromb. Haemost. 89:747-751.

11. Eitzman, D.T., et al. 2005. Homozygosity for factor $V$ Leiden leads to enhanced thrombosis and atherosclerosis in mice. Circulation. 111:1822-1825.
12. Westrick, R.J., et al. 2001. Deficiency of tissue factor pathway inhibitor promotes atherosclerosis and thrombosis in mice. Circulation. 103:3044-3046.

13. Buchanan, M.R., and Brister, S.J. 1998. Inhibition of chronic vessel wall intimal hyperplasia following acute anticoagulant treatment: relative effects of heparin and dermatan sulphate. Thromb. Res. 91:157-167.

14. Aihara, K., et al. 2007. Strain-dependent embryonic lethality and exaggerated vascular remodeling in heparin cofactor II-deficient mice. J. Clin. Invest. 117:1514-1526. doi:10.1172/JCI27095.

15. Tollefsen, D.M. 2007. Heparin cofactor II modulates the response to vascular injury. Arterioscler. Thromb. Vasc. Biol. 27:454-460.

16. Mackman, N. 2004. Mouse models in haemostasis and thrombosis. Thromb. Haemost. 92:440-443.

17. He, L., Vicente, C.P., Westrick, R.J., Eitzman, D.T., and Tollefsen, D.M. 2002. Heparin cofactor II inhibits arterial thrombosis after endothelial injury. J. Clin. Invest. 109:213-219. doi:10.1172/ JCI200213432.
18. Giri, T.K., Ahn, C.W., Wu, K.K., and Tollefsen, D.M. 2005. Heparin cofactor II levels do not predict the development of coronary heart disease: the Atherosclerosis Risk in Communities (ARIC) study. Arterioscler. Thromb. Vasc. Biol. 25:2689-2690.

19. Schillinger, M., et al. 2004. High plasma heparin cofactor II activity protects from restenosis after femoropopliteal stenting. Thromb. Haemost. 92:1108-1113.

20. Takamori, N., et al. 2004. High plasma heparin cofactor II activity is associated with reduced incidence of in-stent restenosis after percutaneous coronary intervention. Circulation. 109:481-486.

21. Chackalamannil, S. 2006. Thrombin receptor (protease activated receptor-1) antagonists as potent antithrombotic agents with strong antiplatelet effects. J. Med. Chem. 49:5389-5403.

22. Schering-Plough Corp. 2007. Schering-Plough's novel oral thrombin receptor antagonist meets primary endpoint in phase II trial [news release]. http:// www.schering-plough.com/schering_plough/news/ release.jsp?releaseID $=977603$. 